\title{
The crystal structure of natrolite*
}

\author{
By W. M. MEIER \\ Gates and Crellin Laboratories, California Institute of Technology \\ Pasadena, California
}

With 5 figures

(Received September 4, 1959)

\begin{abstract}
Auszug
Eine genaue Strukturbestimmung von Natrolith, $\mathrm{Na}_{2} \mathrm{Al}_{2} \mathrm{Si}_{3} \mathrm{O}_{10} \cdot 2 \mathrm{H}_{2} \mathrm{O}$, mittels Fourier-Synthesen und der Methode der kleinsten Quadrate führte zu einer Bestätigung der von PAULING und TAYLOR vorgeschlagenen Struktur. Wasserstoffbrücken von 2,85 bzw. 2,99 $\AA$ Länge verbinden die Wassermoleküle mit Sauerstoffatomen des Alumosilikatgerüstes. Die Strukturbestimmung lieferte folgende Atomabstände: $1,62 \pm 0,01 \AA$ für die tetraedrische Si-OBindung, 1,75 $\pm 0,02 \AA$ für die tetraedrische $\mathrm{Al}-\mathrm{O}$-Bindung und 2,37 $\pm 0,02 \AA$ für die $\mathrm{Na}-\mathrm{O}$-Bindung.

Die Öfnungen zwischen benachbarten Ketten der Faserzeolithe formen ziemlich weite Kanäle, die die Struktur durchziehen. Die beobachteten Austauschvorgänge sind auf diese Kanäle zurückzuführen und nicht, wie dies lange vermutet wurde, auf die viel engeren, parallel zur Faserachse verlaufenden Kanäle.
\end{abstract}

\section{Abstract}

The structure of natrolite, $\mathrm{Na}_{2} \mathrm{Al}_{2} \mathrm{Si}_{3} \mathrm{O}_{10} \cdot 2 \mathrm{H}_{2} \mathrm{O}$, proposed by PAULING and TAYLOR has been confirmed and refined by Fourier and least-squares methods using three-dimensional data obtained with $\mathrm{CuK} \alpha$ radiation. The water is linked to oxygen atoms of the framework by hydrogen bonds having a length of 2.85 and $2.99 \AA$, respectively. The refined structure gives interatomic distances of $1.62 \pm 0.01 \AA$ for the tetrahedral Si-O bond, $1.75 \pm 0.02 \AA$ for the tetrahedral AI- $\mathrm{O}$ bond, and $2.37 \pm 0.02 \AA$ for the $\mathrm{Na}-\mathrm{O}$ bond.

The openings between neighboring chains of the fibrous zeolites provide a system of open, intersecting channels. The dehydration and cation exchange phenomena must be attributed to these channels and not, as has long been supposed, to the much narrower channels parallel to the fiber axis.

* Contribution No. 2485 from the Gates and Crellin Laboratories of Chemistry. 


\section{Introduction}

The general features of many zeolite structures are sufficiently well known to provide possible explanations of their molecular-sieve and cation-exchange properties. More precise information about these structures is needed, however, to gain a full understanding of the nature of these porous crystals. Natrolite, a representative member of the fibrous zeolites, appeared to be well suited for a precise x-ray investigation with modern methods in order to further our knowledge of zeolite structures.

The structure of natrolite, $\mathrm{Na}_{2} \mathrm{Al}_{2} \mathrm{Si}_{3} \mathrm{O}_{10} \cdot 2 \mathrm{H}_{2} \mathrm{O}$, was first proposed by Pauling (1930). It consists of characteristic chains of $\mathrm{AlO}_{4}$ and

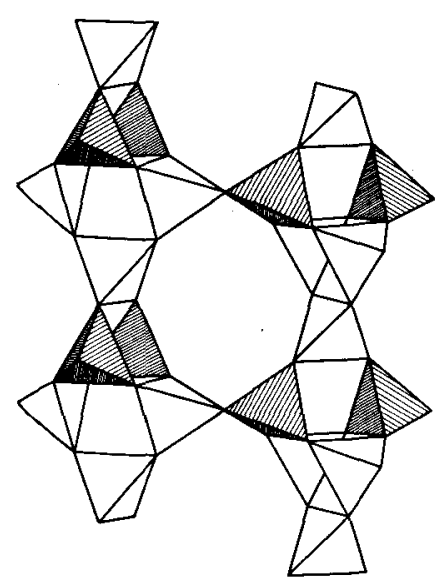

Fig. 1

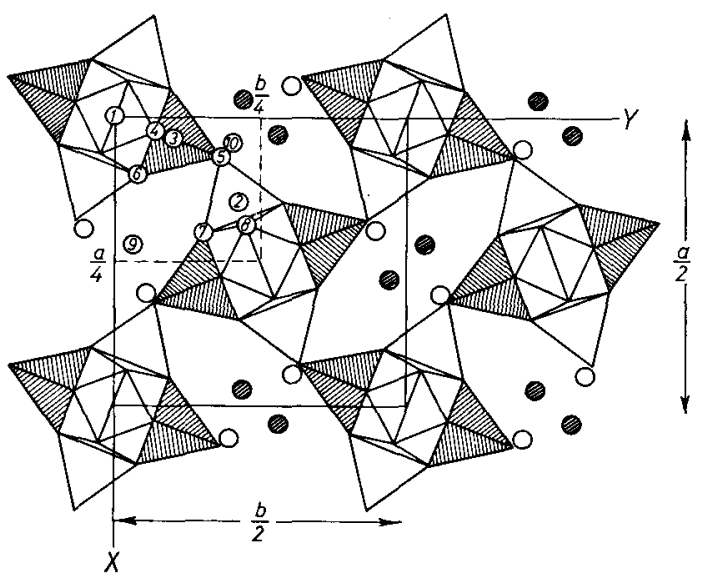

Fig. 2

Fig. 1. The chains in natrolite. The lower $\mathrm{SiO}_{4}$ tetrahedra of a chain are linked to the upper $\mathrm{AlO}_{4}$ tetrahedra (shaded) of neighboring chains.

Fig. 2. (001) projection of the natrolite structure. The repeat distances are $a / 2$ and $b / 2$ in this projection. The $\mathrm{AlO}_{4}$ tetrahedra have been shaded. The notation used for the atoms of the asymmetric unit is as follows:
1. $\mathrm{Si}_{\mathrm{I}}$
3. $\mathrm{Al}$
5. $\mathrm{O}_{\text {II }}$
7. $\mathrm{O}_{\mathrm{IV}}$
9. $\mathrm{Na}$
2. $\mathrm{Si}_{\mathrm{II}}$
4. $\mathrm{O}_{\mathrm{I}}$
6. $\mathrm{O}_{\mathrm{III}}$
8. $\mathrm{O}_{\mathrm{V}}$
10. $\mathrm{H}_{2} \mathrm{O}$

$\mathrm{SiO}_{4}$ tetrahedra which are linked together in the way shown in Figs. 1 and 2. TAYLOR, MEEK and JACKSON (1933) subsequently determined the approximate locations of all the atoms in the structure. They calculated structure factors for a number of reflections and found good agreement with the observed values ${ }^{1}$. This seemed to indicate that the

1 The atomic coordinates given by TAYLOR et al. give a disagreement factor of 0.31 for the observable $h k 0$ reflections. 
structure was essentially correct. It was also demonstrated by TAYLOR (1934) that the chains found in natrolite can be linked in three different ways to give the structures of natrolite, thomsonite, and edingtonite.

\section{Experimental work}

The natrolite crystals used in this investigation were perfectly clear prisms of about $0.1 \mathrm{~mm}$ thickness from Aussig, Bohemia. Their composition was checked by a flamespectrometric determination of the cation content $^{2}$. The analysis gave $15.8 \pm 0.3 \% \mathrm{Na}_{2} \mathrm{O}$, which is only slightly below the expected value of $16.3 \%$ based on the formula. In addition $0.04 \% \mathrm{~K}_{2} \mathrm{O}$ was found but no other cations such as calcium could be detected.

\section{Unit cell and space group}

The unit cell of natrolite is orthorhombic and contains 8 formula units of $\mathrm{Na}_{2} \mathrm{Al}_{2} \mathrm{Si}_{3} \mathrm{O}_{10} \cdot 2 \mathrm{H}_{2} \mathrm{O}$ according to previous workers. Accurate cell dimensions were obtained from rotation photographs of the Straumanis type with the crystal rotating around the $c$ axis. Values of $a$ and $b$ were calculated from high-angle reflections and the results plotted against $\sin ^{2} \theta$ to allow for absorption and other corrections. The cell constant $c$ was determined by means of the layer-line spacings. The cell dimensions obtained

$$
\begin{aligned}
& a=18.30 \pm 0.01 \AA \\
& b=18.63 \pm 0.01 \AA \\
& c=6.60 \pm 0.02 \AA
\end{aligned}
$$

are in agreement with the established values of $a=18.3, b=18.6$, and $c=6.6 \AA$. A different specimen of natrolite from Tick Canyon, California, gave the same cell constants within the limits of error.

The observed reflections clearly indicate a face-centered lattice, and the absence of reflections $h 0 l$ and $0 k l$ with $h+l \neq 4 n$ and $k+l \neq 4 n$, respectively, defines the space group as $C_{2 v}^{19}-F d d 2$. A weak spot corresponding to the forbidden reflection 402 gave rise to some doubt as to the correct space group in earlier investigations (Halla and Merl, 1930; Hey and Bannister, 1933). This weak reflection has also been observed and has been identified as a double reflection from the planes $\overline{\mathbf{1}} 11$ and $\overline{\mathbf{5}} \overline{\mathbf{1}} 1$ (Renninger effect).

${ }^{2}$ I am grateful to Mr. A. A. Chodos, Division of Geological Sciences, California Institute of Technology, for practical assistance in the determination. 
Intensity measurements

Intensities were obtained from equi-inclination Weissenberg photographs around [001] using filtered $\mathrm{Cu}$ radiation. A full set of threedimensional data was collected using a small crystal of $0.08 \mathrm{~mm}$ thickness and $0.3 \mathrm{~mm}$ length. The use of the triple-film technique and long and short exposures produced an intensity range of about 1 to 6000 . The intensities were estimated visually by comparison with standard intensity scales prepared with the same crystal. Only 15 of the 300 possible reflections with even indices were found to have intensities below the limit of observation. The intensity values were corrected for Lorentz and polarization factors in the usual way. Corrections for absorption effects were not applied.

The observed structure factors were initially placed on an absolute scale by the method of WILsoN, which also provided a provisional temperature factor of $1.1 \AA^{2}$. The scaling factors were further adjusted in the course of the refinement by comparison with the calculated structure factors.

\section{Verification and refinement of the strueture Procedure}

The testing and first refinement of the structure were carried out with the use of the $h k 0$ data because of the centrosymmetry of the (001) projection. The subsequent refinement in three dimensions was largely determined by the fact that the structure factors for reflections of the even layers can be reduced to

and

$$
\left.\begin{array}{l}
A=16 \cos 2 \pi h x \cos 2 \pi k y \cos 2 \pi l z \\
B=16 \cos 2 \pi h x \cos 2 \pi k y \sin 2 \pi l z
\end{array}\right\} \text { for } h+k+l=4 n
$$

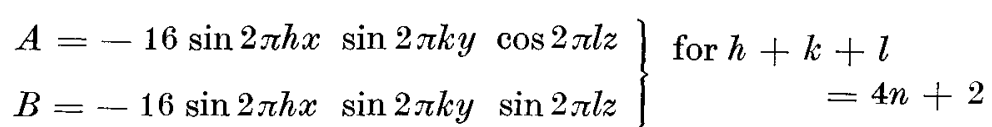

whereas the corresponding equations for reflections of the odd layers are considerably more complicated. The systematic refinement in three dimensions was therefore carried out by least-squares methods using the data from the even layers $(h k 0, h k 2$, and $h k 4)$ and the weighting system described by HuGHEs (1941). Structure factors were finally calculated for the $h k 1$ reflections to provide a check for the correctness of the $z$ parameters which, strictly speaking, cannot be determined unambiguously on the basis of the even layers alone. 
Hand calculations in the early stages of the refinement were followed by the use of the Datatron 205 electronic computer, at the California Institute of Technology, for the computation of Fourier syntheses, structure factors and least-squares parameter adjustments. The atomic scattering factors used in this study were those calculated by BERGHUIs et al. (1955) for $\mathrm{Na}$ and $\mathrm{O}$, and ToMHE and StAMr (1958) for Si and Al.

\section{Testing of the structure}

The positional parameters for the trial structure were derived on the basis of regular $(\mathrm{Al}, \mathrm{Si}) \mathrm{O}_{4}$ tetrahedra of average dimension, and atomic scattering factors for $\mathrm{Si}$ were used in the beginning for both $\mathrm{Si}$ and $\mathrm{Al}$ positions in order to avoid any initial assumption about the $\mathrm{Al}-\mathrm{Si}$ distribution. The positional parameters of the trial structure differed somewhat from the atomic coordinates given by TAXLOR, MEEK and $J_{\text {ACKSON (1933). Calculated and observed structure factors }}$ for $h k 0$ gave an $R_{1}$ factor $^{3}$ of 0.37 for the 96 observable reflections, and provided the signs for 68 reflections. A Fourier synthesis using these reflections confirmed the general features of the trial structure and suggested some parameter changes. After three stages of refinement by means of $F_{o}$ and $\left(F_{o}-F_{c}\right)$ Fourier syntheses it was evident that the $\mathrm{SiO}_{4}$ and $\mathrm{AlO}_{4}$ tetrahedra are ordered in the way proposed by PaULing and TAYLOR. Definite sites were accordingly assigned to the $\mathrm{Al}$ and $\mathrm{Si}$ atoms in the subsequent refinement.

\section{Two-dimensional refinement}

Two more refinement cycles by means of difference syntheses and the use of individual isotropic temperature factors lowered $R_{1}$ to 0.214 . Further refinement was achieved by least-squares calculations using all the $h k 0$ data. Individual isotropic temperature parameters were thereby used and shifts were also applied to the scaling factor. The $R_{1}$ factor was lowered to 0.077 in the course of seven such refinement cycles. The positional and temperature parameters obtained in this way are listed in Table 1.

\section{Three-dimensional refinement}

The three-dimensional refinement was also accomplished by means of the least-squares method. The $h k 2$ and $h k 4$ data were first refined independently in order to obtain sufficiently reliable values for the

${ }^{3}$ Defined as $\sum\left|F_{o}\right|-\left|F_{\mathrm{c}}\right| / \sum\left|\boldsymbol{F}_{0}\right|$. 
Table 1. Parameters obtained by the separate refinement of the layer-line data

\begin{tabular}{l|c|c|c}
\hline & $\begin{array}{c}(h k 0) \\
\text { refinement }\end{array}$ & $\begin{array}{c}(h k 2) \\
\text { refinement }\end{array}$ & $\begin{array}{c}(h k 4) \\
\text { refinement }\end{array}$ \\
\hline
\end{tabular}

(a) Positional parameters and $R$ values

\begin{tabular}{|c|c|c|c|c|}
\hline $\mathrm{Si}_{\mathrm{II}}$ & $\begin{array}{l}x_{2} \\
y_{2} \\
z_{2}\end{array}$ & $\begin{array}{l}0.1531 \\
0.2114\end{array}$ & $\begin{array}{l}0.1535 \\
0.2112 \\
0.6192\end{array}$ & $\begin{array}{l}0.1537 \\
0.2116 \\
0.6172\end{array}$ \\
\hline $\mathrm{Al}$ & $\begin{array}{l}x_{3} \\
y_{3} \\
z_{3}\end{array}$ & $\begin{array}{l}0.0375 \\
0.0937\end{array}$ & $\begin{array}{l}0.0375 \\
0.0934 \\
0.6108\end{array}$ & $\begin{array}{l}0.0372 \\
0.0934 \\
0.6118\end{array}$ \\
\hline $\mathrm{O}_{\mathrm{I}}$ & $\begin{array}{l}x_{4} \\
y_{4} \\
z_{4}\end{array}$ & $\begin{array}{l}0.0225 \\
0.0682\end{array}$ & $\begin{array}{l}0.0245 \\
0.0688 \\
0.8508\end{array}$ & $\begin{array}{l}0.0234 \\
0.0693 \\
0.8542\end{array}$ \\
\hline$O_{i \mathrm{I}}$ & $\begin{array}{l}x_{5} \\
y_{5} \\
z_{5}\end{array}$ & $\begin{array}{l}0.0690 \\
0.1840\end{array}$ & $\begin{array}{l}0.0712 \\
0.1823 \\
0.6028\end{array}$ & $\begin{array}{l}0.0700 \\
0.1826 \\
0.6004\end{array}$ \\
\hline $\mathrm{O}_{\mathrm{III}}$ & $\begin{array}{l}x_{6} \\
y_{6} \\
z_{6}\end{array}$ & $\begin{array}{l}0.0987 \\
0.0346\end{array}$ & $\begin{array}{l}0.0982 \\
0.0345 \\
0.4965\end{array}$ & $\begin{array}{l}0.0987 \\
0.0356 \\
0.4990\end{array}$ \\
\hline $\mathrm{O}_{\mathrm{IV}}$ & $\begin{array}{l}x_{7} \\
y_{7} \\
z_{7}\end{array}$ & $\begin{array}{l}0.2060 \\
0.1529\end{array}$ & $\begin{array}{l}0.2059 \\
0.1525 \\
0.7270\end{array}$ & $\begin{array}{l}0.2064 \\
0.1523 \\
0.7232\end{array}$ \\
\hline$O_{V}$ & $\begin{array}{l}x_{8} \\
y_{8} \\
z_{8}\end{array}$ & $\begin{array}{l}0.1803 \\
0.2266\end{array}$ & $\begin{array}{l}0.1782 \\
0.2277 \\
0.3861\end{array}$ & $\begin{array}{l}0.1778 \\
0.2283 \\
0.3847\end{array}$ \\
\hline $\mathrm{Na}$ & $\begin{array}{l}x_{9} \\
y_{9} \\
z_{9}\end{array}$ & $\begin{array}{l}0.2209 \\
0.0302\end{array}$ & $\begin{array}{l}0.2206 \\
0.0311 \\
0.6107\end{array}$ & $\begin{array}{l}0.2211 \\
0.0308 \\
0.6123\end{array}$ \\
\hline $\mathrm{H}_{2} \mathrm{O}$ & $\begin{array}{l}x_{10} \\
y_{10} \\
z_{10} \\
R\end{array}$ & $\begin{array}{l}0.0593 \\
0.1874 \\
7.7 \%\end{array}$ & $\begin{array}{l}0.0579 \\
0.1889 \\
0.1105 \\
7.0 \%\end{array}$ & $\begin{array}{l}0.0571 \\
0.1886 \\
0.1100 \\
9.4 \%\end{array}$ \\
\hline
\end{tabular}

(b) Thermal parameters $B$ (in $\AA^{2}$ )

\begin{tabular}{l|}
$\mathrm{Si}_{I}$ \\
$\mathrm{Si}_{I I}$ \\
$\mathrm{Al}$ \\
$\mathrm{O}_{\mathrm{I}}$ \\
$\mathrm{O}_{\mathrm{II}}$ \\
$\mathrm{O}_{\mathrm{III}}$ \\
$\mathrm{O}_{\mathrm{IV}}$ \\
$\mathrm{O}_{\mathrm{V}}$ \\
$\mathrm{Na}$ \\
$\mathrm{H}_{2} \mathrm{O}$
\end{tabular}

1.0
0.8
0.7
1.5
1.4
1.3
1.0
1.5
1.7
2.9


respective scaling factors. Four cycles of refinements were carried out for each layer, and the disagreement factors attained were $R_{2}=0.070$ for $h k 2$ and $R_{3}=0.094$ for $h k 4$. The results in Table 1 show satisfactory agreement, on the whole, between the positional parameters derived from the different layers. The discrepancies in the temperature parameters, if significant at all, seem to indicate errors in the scaling, and possibly some anisotropy in the thermal vibrations.

The data from the even layers were eventually combined for the final least-squares refinement. A total of 9 very strong reflections in the small-angle region were left out in this refinement since they appeared to be strongly affected by absorption or extinction. Average values for the parameters (from Table 1) lead to an $R$ factor of 0.090 for the 284 observable reflections with even indices. Six stages of refinement eventually lowered $R$ to 0.083 . The last cycle did not indicate any

Table 2. Final parameters

\begin{tabular}{|c|c|c|c|c|c|c|c|}
\hline \multirow[t]{2}{*}{ Atom } & \multicolumn{3}{|c|}{$\begin{array}{l}\text { Final atomic coordinates } \\
\text { (With standard deviations) }\end{array}$} & \multirow{2}{*}{$\begin{array}{c}B \\
\text { values } \\
\left.\text { (in } \AA^{2}\right)\end{array}$} & \multicolumn{3}{|c|}{$\begin{array}{c}\text { Corresponding } \\
\text { coordinates given by } \\
\text { TAYLOR et al. (1933) }\end{array}$} \\
\hline & $x$ & $y$ & $z$ & & $x$ & $y$ & $z$ \\
\hline $\mathrm{Si}_{\mathrm{I}}$ & 0 & 0 & 0 & 1.07 & 0 & 0 & 0 \\
\hline$S i_{\text {II }}$ & $\begin{array}{c}0.1532 \\
(0.0002)\end{array}$ & $\begin{array}{c}0.2112 \\
(0.0002)\end{array}$ & $\begin{array}{c}0.6181 \\
(0.0010)\end{array}$ & 1.00 & 0.153 & 0.208 & 0.625 \\
\hline $\mathrm{Al}$ & $\begin{array}{c}0.0376 \\
(0.0002)\end{array}$ & $\begin{array}{c}0.0936 \\
(0.0002)\end{array}$ & $\begin{array}{c}0.6119 \\
(0.0011)\end{array}$ & 1.12 & 0.036 & 0.089 & 0.625 \\
\hline $\mathrm{O}_{\mathrm{I}}$ & $\begin{array}{c}0.0227 \\
(0.0004)\end{array}$ & $\begin{array}{c}0.0683 \\
(0.0004)\end{array}$ & $\begin{array}{c}0.8594 \\
(0.0024)\end{array}$ & 1.81 & 0.014 & 0.067 & 0.875 \\
\hline $\mathrm{O}_{\mathrm{II}}$ & $\begin{array}{c}0.0704 \\
(0.0004)\end{array}$ & $\begin{array}{c}0.1824 \\
(0.0003)\end{array}$ & $\begin{array}{c}0.6011 \\
(0.0023)\end{array}$ & 1.10 & 0.069 & 0.181 & 0.625 \\
\hline $\mathrm{O}_{\text {III }}$ & $\begin{array}{c}0.0986 \\
(0.0004)\end{array}$ & $\begin{array}{c}0.0346 \\
(0.0004)\end{array}$ & $\begin{array}{c}0.4997 \\
(0.0020)\end{array}$ & 1.55 & 0.097 & 0.042 & 0.500 \\
\hline $\mathrm{O}_{\mathrm{IV}}$ & $\begin{array}{c}0.2063 \\
(0.0002)\end{array}$ & $\begin{array}{c}0.1526 \\
(0.0004)\end{array}$ & $\begin{array}{c}0.7166 \\
(0.0022)\end{array}$ & 1.24 & 0.208 & 0.153 & 0.750 \\
\hline $\mathrm{O}_{\mathrm{v}}$ & $\begin{array}{c}0.1799 \\
(0.0002)\end{array}$ & $\begin{array}{c}0.2272 \\
(0.0004)\end{array}$ & $\begin{array}{c}0.3860 \\
(0.0020)\end{array}$ & 1.83 & 0.183 & 0.236 & 0.375 \\
\hline $\mathrm{Na}$ & $\begin{array}{c}0.2208 \\
(0.0003)\end{array}$ & $\begin{array}{c}0.0305 \\
(0.0002)\end{array}$ & $\begin{array}{c}0.6120 \\
(0.0016)\end{array}$ & 2.00 & 0.222 & 0.028 & 0.625 \\
\hline $\mathrm{H}_{2} \mathrm{O}$ & $\begin{array}{c}0.0564 \\
(0.0005)\end{array}$ & $\begin{array}{c}0.1893 \\
(0.0004)\end{array}$ & $\begin{array}{c}0.1085 \\
(0.0038)\end{array}$ & 2.50 & 0.069 & 0.181 & 0.125 \\
\hline
\end{tabular}


further decrease in the inaccuracy total $\Sigma w\left(F_{o}-F_{c}\right)^{2}$. The scaling factors of the individual layers were still subject to small adjustments in the last stages of the refinement. The final parameters are given in Table 2 as compared with the values found by TAYLOR.

Comparison of observed $h k 1$ structure factors with the calculated values using the final parameters showed good agreement (Table 3) and resulted in a disagreement factor of 0.077 for the 103 observable reflections in the first layer line. The correctness of the $z$ coordinates could thus be confirmed.

An $h k 0$ Fourier synthesis based on the signs calculated from the final parameters is shown in Fig. 3.

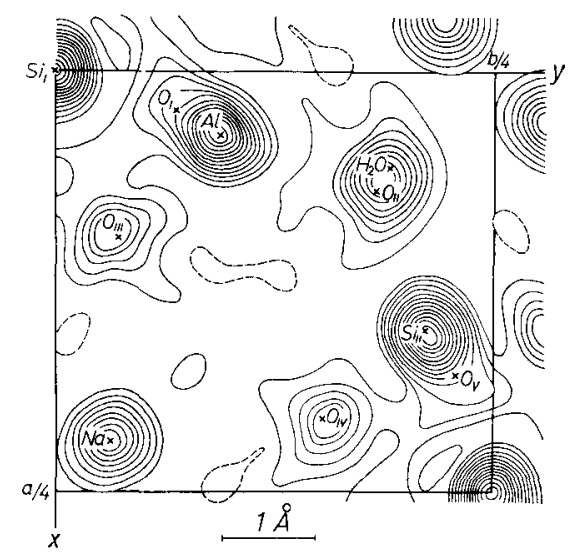

Fig. 3. Electron density projection along [001]. The contours are at intervals of 200 units $=2.35 \mathrm{e}^{-2}$ starting with zero (broken line).

\section{Results}

Standard deviations $\sigma$ for the final atomic coordinates were calculated from the least-squares totals obtained in the last refinement cycle. They are listed with the coordinates in Table 2. The accuracy of the atomic positions of most atoms is around $0.005 \AA$ for the $x$ and $y$ coordinates and somewhat over $0.01 \AA$ for the $z$ coordinates. The coordinates of the water molecules are by far the least accurate, with a $\sigma$-value of about $0.01 \AA$ for $x$ and $y$, and $0.025 \AA$ for $z$.

Table 4 contains interatomic distances and bond angles together with estimated standard deviations. The standard deviations for the bond angles have values between $1^{\circ}$ and $2^{\circ}$. 
W. M. MEIER

Table 3. Observed and calculated structure factors

\begin{tabular}{|c|c|c|c|c|c|c|c|c|c|c|c|c|c|c|}
\hline h & k 2 & $\left|F_{0}\right|$ & $\mathbf{F}_{\mathrm{c}}$ & b & k 1 & $\left|F_{a}\right|$ & $\mathbf{F}_{\mathrm{c}}$ & $b k 1$ & $\left|F_{0}\right|$ & $\left|\mathrm{F}_{\mathrm{c}}\right|$ & h & \& 1 & $\left|F_{0}\right|$ & $\left|P_{\mathrm{c}}\right|$ \\
\hline 0 & 40 & 201 & 241 & 10 & 180 & 31 & 25 & 1211 & 14 & 20 & 13 & 31 & 91 & 91 \\
\hline 0 & 80 & 186 & -176 & 10 & 200 & 24 & 14 & 1251 & 60 & 50 & 13 & 51 & 143 & 153 \\
\hline & 120 & 29 & -25 & & & & & & & & 13 & 71 & 55 & 58 \\
\hline 0 & 160 & 162 & -166 & 12 & 00 & 39 & -38 & $\begin{array}{lll}3 & 1 & 1\end{array}$ & 156 & 180 & 13 & 91 & 112 & 97 \\
\hline 0 & 200 & 40 & 36 & 12 & 20 & 30 & 26 & $3 \quad 31$ & 44 & 42 & 131 & 111 & 87 & 75 \\
\hline \multirow{2}{*}{\multicolumn{2}{|c|}{0240}} & 21 & 28 & 12 & 40 & 181 & 194 & 351 & 271 & 314 & 131 & 131 & 79 & 78 \\
\hline & & & & 12 & 60 & 118 & 121 & 371 & 69 & 58 & 131 & 151 & 48 & 45 \\
\hline 2 & 20 & 166 & 191 & 12 & 80 & 28 & 26 & 391 & 64 & 60 & 131 & 171 & $<14$ & 12 \\
\hline 2 & 40 & 165 & 178 & 12 & 100 & 50 & 42 & 3111 & 82 & 82 & 131 & $19 \mathrm{I}$ & 22 & 19 \\
\hline 2 & 60 & 92 & -90 & 12 & 120 & 61 & -58 & 3131 & 81 & 80 & & & & \\
\hline 2 & 80 & 74 & 79 & 12 & 140 & 64 & -63 & 3151 & 74 & 76 & 15 & 11 & 156 & 184 \\
\hline & 100 & 232 & 239 & 12 & 160 & 101 & 98 & 3171 & $<18$ & 23 & 15 & 31 & 93 & 92 \\
\hline 21 & 120 & 56 & -52 & 12 & 180 & 34 & -29 & 3191 & 36 & 38 & 15 & 51 & 70 & 66 \\
\hline 21 & 140 & $<10$ & -12 & 12 & 200 & 31 & -23 & 3211 & 63 & 54 & 15 & $7 \mathrm{t}$ & 67 & 57 \\
\hline 21 & 160 & 43 & -35 & & & & & 3231 & 13 & 13 & 15 & 91 & 75 & 78 \\
\hline 21 & 180 & $<11$ & 12 & 14 & 20 & 36 & -35 & & & & 15 & 111 & 89 & 86 \\
\hline 22 & 200 & $<10$ & 3 & 14 & 40 & 58 & -53 & 5111 & 199 & 219 & 15 & 131 & 39 & 33 \\
\hline \multirow{2}{*}{\multicolumn{2}{|c|}{2220}} & 117 & 104 & 14 & 60 & 218 & 232 & 531 & 272 & 299 & 15 & 151 & 53 & 47 \\
\hline & & & & 14 & 80 & 29 & 28 & 551 & 10 & 12 & 15 & 171 & 52 & 42 \\
\hline 4 & $\begin{array}{lll}0 & 0\end{array}$ & 170 & 194 & 14 & 100 & 16 & 14 & 571 & 29 & 28 & & & & \\
\hline 4 & 20 & 143 & -136 & 14 & 120 & 15 & 19 & 5991 & 106 & 98 & 17 & 11 & 89 & 97 \\
\hline 4 & 40 & 92 & -88 & 14 & 140 & 71 & 62 & 5111 & 26 & 23 & 17 & 31 & $<18$ & 11 \\
\hline 4 & 60 & 55 & 54 & 14 & 160 & 43 & -36 & 5131 & 137 & 149 & 17 & 51 & 92 & 86 \\
\hline 4 & 80 & 72 & 65 & 14 & 180 & 36 & 29 & 5151 & 71 & 69 & 17 & 71 & 52 & 46 \\
\hline 41 & 100 & 251 & 283 & & & & & 5171 & 81 & 77 & 17 & 91 & 57 & 59 \\
\hline 41 & 120 & 201 & 203 & 16 & 00 & 161 & -155 & 5191 & 23 & 33 & 17 & 111 & 43 & 34 \\
\hline 41 & 140 & 35 & 33 & 16 & 20 & $<11$ & -7 & 5211 & 57 & 50 & 171 & 131 & 30 & 25 \\
\hline 41 & 160 & 40 & 40 & 16 & 40 & $<11$ & 15 & 5231 & 51 & 55 & 17 & 151 & 48 & 45 \\
\hline 41 & 180 & 66 & -57 & 16 & 60 & $<11$ & -7 & & & & & & & \\
\hline 42 & 200 & 50 & 45 & 16 & 80 & 58 & 57 & 711 & 190 & 192 & 19 & 11 & 37 & 41 \\
\hline \multirow{2}{*}{\multicolumn{2}{|c|}{4220}} & 64 & -51 & 16 & 100 & 33 & 31 & 731 & 52 & 42 & 19 & 31 & $<16$ & 14 \\
\hline & & & & 16 & 120 & 106 & 98 & 751 & 25 & 26 & 19 & 51 & 23 & 27 \\
\hline 6 & 20 & 111 & -111 & 16 & 140 & 45 & 38 & 771 & 145 & 152 & 19 & 71 & 46 & 38 \\
\hline 6 & 40 & 70 & -75 & 16 & 160 & 167 & 162 & 791 & 117 & 109 & 19 & 91 & 81 & so \\
\hline 6 & 60 & 169 & -161 & & & & & 7111 & 62 & 61 & 191 & 111 & 66 & 67 \\
\hline 6 & 80 & 24 & -19 & 18 & 20 & 45 & 42 & 7131 & 17 & 28 & 19 & 131 & 34 & 32 \\
\hline 61 & 100 & 133 & 125 & 18 & 40 & 19 & 15 & 7151 & 76 & 71 & & & & \\
\hline 61 & 120 & 91 & -84 & 18 & 60 & 112 & 101 & 7171 & 55 & 50 & 21 & 11 & 27 & 27 \\
\hline 61 & 140 & 254 & 261 & 18 & 80 & 67 & -60 & 7191 & 49 & 47 & 21 & 31 & 66 & 60 \\
\hline 61 & 160 & $<11$ & 11 & 18 & 100 & 24 & 21 & 7211 & 55 & 61 & 21 & 51 & 44 & 45 \\
\hline 61 & 180 & 127 & 121 & 18 & 120 & 23 & 19 & & & & 21 & 71 & 50 & 53 \\
\hline 62 & 200 & 101 & 89 & 18 & 140 & $<6$ & -4 & $\begin{array}{lll}9 & 1 & 1\end{array}$ & 73 & 64 & 21 & 91 & 16 & 20 \\
\hline \multirow{2}{*}{\multicolumn{2}{|c|}{622}} & 14 & -9 & & & & & 931 & 57 & 53 & & & & \\
\hline & & & & 20 & 00 & 70 & 66 & $\begin{array}{lll}9 & 5 & 1\end{array}$ & 104 & 102 & 23 & 11 & 33 & 40 \\
\hline $\mathrm{s}$ & 00 & 162 & -151 & 20 & 20 & $<10$ & 5 & 971 & 118 & 121 & 23 & 31 & 27 & 33 \\
\hline $\mathrm{s}$ & 20 & 95 & -101 & 20 & 40 & 48 & 44 & 991 & 189 & 193 & & & & \\
\hline 8 & 40 & 105 & 107 & 20 & 60 & 71 & -60 & 9111 & 98 & 102 & 0 & 22 & 145 & 175 \\
\hline 8 & 60 & $<B$ & -5 & 20 & 80 & 28 & 26 & 9131 & 116 & 113 & 0 & 62 & 163 & 170 \\
\hline 8 & 80 & 107 & 103 & 20 & 100 & 16 & -14 & 9151 & 108 & 100 & 0 & 102 & 237 & 263 \\
\hline 81 & 100 & 89 & 83 & 20 & 120 & 34 & -28 & 9171 & 78 & 68 & o & 142 & 92 & 91 \\
\hline 81 & 120 & $<10$ & -8 & & & & & 9191 & 89 & 89 & o & 182 & 78 & 85 \\
\hline 8 & 140 & $<11$ & -3 & 22 & 20 & 100 & 93 & 9211 & 29 & 26 & o & 222 & 66 & 60 \\
\hline 81 & 160 & 45 & 41 & 22 & 40 & 72 & 72 & & & & & & & \\
\hline 81 & 180 & 72 & 65 & 22 & 60 & 10 & -8 & $11 \quad 11$ & 146 & 157 & 2 & 02 & 158 & 192 \\
\hline 8 & 200 & 29 & 26 & 22 & 80 & 5 & 4 & 1131 & 99 & 103 & 2 & 22 & 184 & 224 \\
\hline \multirow[t]{2}{*}{822} & 220 & 10 & 1 & & & & $\left|\mathbf{F}_{\mathrm{c}}\right|$ & $\begin{array}{lll}1151\end{array}$ & 15 & 18 & 2 & 42 & 126 & 123 \\
\hline & & & & 1 & 31 & 155 & 166 & 1171 & 64 & 60 & 2 & 62 & 170 & 194 \\
\hline 10 & 20 & 227 & 238 & 1 & 51 & 197 & 200 & 1191 & 103 & 99 & 2 & 82 & 63 & 59 \\
\hline 10 & 40 & 257 & -287 & 1 & 71 & 181 & 191 & 11111 & 121 & 129 & 2 & 102 & 50 & .47 \\
\hline 10 & 60 & 149 & 153 & 1 & 91 & 79 & 74 & 11131 & 98 & 95 & 2 & 122 & 142 & 149 \\
\hline 10 & 80 & 58 & -51 & & 111 & 147 & 156 & $\begin{array}{lll}11 & 151\end{array}$ & 67 & 54 & 2 & 142 & 20 & 19 \\
\hline 10 & 100 & 185 & -192 & 1 & 131 & 74 & 72 & 11171 & 46 & 42 & 2 & 162 & 17 & 18 \\
\hline 10 & 120 & 46 & -33 & 1 & 151 & 173 & 185 & 11191 & 76 & 71 & 2 & 182 & 94 & 98 \\
\hline 10 & 140 & 19 & -12 & 1 & 171 & 126 & 127 & & & & & 202 & 73 & 73 \\
\hline 10 & 160 & 44 & -37 & 1 & 191 & $<17$ & 17 & 1311 & 64 & 71 & 2 & 222 & 44 & 41 \\
\hline
\end{tabular}


Table 3 (continued)

\begin{tabular}{|c|c|c|c|c|c|c|c|c|c|c|c|c|c|}
\hline b 12 & $\left|P_{0}\right|$ & $\left|F_{\mathrm{e}}\right|$ & b & 11 & $\left|\mathbf{F}_{0}\right|$ & $\left|P_{\mathrm{e}}\right|$ & h $\leqslant 1$ & $\left|P_{0}\right|$ & $\left|p_{c}\right|$ & . & $\times 1$ & $\left|F_{0}\right|$ & $\left|P_{e}\right|$ \\
\hline 422 & 144 & 158 & 12 & 22 & 103 & 105 & 044 & 28 & 24 & & $16+$ & 53 & 46 \\
\hline 442 & 130 & 120 & 12 & 42 & 101 & 111 & 0 84 & 173 & 192 & & 184 & 97 & 106 \\
\hline 62 & 40 & 38 & 12 & 62 & 112 & 117 & 0124 & 76 & 80 & & & & \\
\hline 482 & 148 & 161 & 12 & 82 & 34 & 38 & 0164 & 107 & 115 & 10 & 24 & 40 & 41 \\
\hline 4102 & 113 & 108 & 12 & 102 & 16 & 12 & 0204 & 51 & 43 & 10 & 44 & 188 & 200 \\
\hline 4122 & 96 & 106 & 12 & 122 & 10 & 8 & & & & 10 & 64 & 44 & 37 \\
\hline 4142 & 142 & 151 & 12 & 142 & 35 & 83 & $\begin{array}{lll}2 & 2 & 4\end{array}$ & 73 & 78 & 10 & 84 & 31 & 26 \\
\hline 4162 & $7^{8}$ & 82 & 12 & 162 & 36 & 35 & 244 & 123 & 129 & 10 & 104 & 126 & 140 \\
\hline 4182 & 79 & 74 & 12 & 182 & 56 & 51 & 264 & 110 & 118 & 10 & 124 & 51 & 43 \\
\hline 4202 & 102 & 111 & & & & & 284 & 170 & 191 & 10 & 144 & 60 & 64 \\
\hline \multirow[t]{2}{*}{4222} & 71 & 68 & 14 & 02 & 112 & 126 & 2104 & 25 & 19 & 10 & 164 & 21 & 18 \\
\hline & & & 14 & 22 & 30 & 30 & 2124 & 52 & 50 & 10 & 184 & 14 & 14 \\
\hline 602 & 132 & 129 & 14 & 42 & 137 & 154 & 2144 & 48 & 46 & & & & \\
\hline 622 & 160 & 177 & 14 & 62 & 71 & 77 & 2164 & 42 & 38 & 12 & 04 & 73 & 77 \\
\hline 642 & 47 & 47 & 14 & 82 & 61 & 58 & 2184 & 50 & 48 & 12 & 24 & 56 & 52 \\
\hline 62 & 39 & 34 & 141 & 102 & 105 & 110 & 2204 & 31 & 29 & 12 & 44 & 16 & 8 \\
\hline 682 & 100 & 103 & 141 & 122 & 96 & 105 & & & & 12 & 64 & 125 & 138 \\
\hline 6102 & 52 & 49 & 14 & 142 & 16 & 13 & 404 & 41 & 38 & 12 & 84 & 50 & 48 \\
\hline 6122 & 103 & 100 & 143 & 162 & 66 & 68 & 424 & 122 & 142 & 12 & 104 & 72 & 71 \\
\hline 6142 & 96 & 91 & 141 & 182 & 50 & 59 & 444 & 80 & 81 & 12 & 124 & 76 & 81 \\
\hline 6162 & 90 & 91 & & & & & 464 & 141 & 155 & 12 & 144 & 44 & 39 \\
\hline 6182 & 57 & 52 & 16 & 22 & 16 & 11 & 484 & 65 & 58 & 12 & 164 & $<6$ & 8 \\
\hline 6202 & 70 & 70 & 16 & 42 & 88 & 105 & 4104 & 193 & 220 & & & & \\
\hline \multirow[t]{2}{*}{6222} & 37 & 36 & 16 & 62 & 84 & 86 & 4124 & 18 & 16 & 14 & 24 & 60 & 64 \\
\hline & & & 16 & 82 & 30 & 31 & 4144 & 34 & 29 & 14 & 44 & 24 & 24 \\
\hline B 22 & 92 & 89 & 161 & 102 & 78 & 78 & 4164 & 58 & 52 & 14 & 64 & 74 & 68 \\
\hline 842 & 130 & 140 & 161 & 122 & 39 & 37 & 4184 & 31 & 26 & 14 & 84 & 38 & 33 \\
\hline 862 & 103 & 104 & 161 & 142 & 59 & 59 & 4204 & 42 & 42 & 14 & 104 & 50 & 43 \\
\hline $8 \quad 82$ & 239 & 282 & 161 & 162 & 14 & 8 & & & & 14 & 124 & 54 & 49 \\
\hline 8102 & 55 & 51 & & & & & $6 \quad 24$ & 105 & 111 & 14 & 144 & 47 & 44 \\
\hline 8122 & 54 & 56 & 18 & 02 & 68 & 69 & 644 & 153 & 173 & & & & \\
\hline 8142 & 77 & 78 & 18 & 22 & 87 & 98 & 664 & 148 & 164 & 16 & 04 & 125 & 137 \\
\hline 8162 & 57 & 53 & 18 & 42 & 61 & 64 & 684 & 36 & 33 & 16 & 24 & 34 & 29 \\
\hline 8182 & 52 & 51 & 18 & 62 & 59 & 64 & 6104 & 18 & 13 & 16 & 44 & 46 & 42 \\
\hline \multirow[t]{2}{*}{8202} & 40 & 39 & 18 & 82 & 39 & 40 & 6124 & 124 & 130 & 16 & 64 & 47 & 49 \\
\hline & & & 181 & 102 & 56 & 58 & 6144 & 70 & 71 & 16 & 84 & 26 & 21 \\
\hline 1002 & 215 & 238 & 181 & 122 & 40 & 41 & 6164 & 34 & 32 & 16 & 104 & $<7$ & 0 \\
\hline 22 & 42 & 38 & 181 & 142 & 42 & 49 & 6184 & 13 & 9 & 16 & 124 & $<6$ & 8 \\
\hline $10 \quad 42$ & 128 & 133 & & & & & 6204 & 91 & 96 & & & & \\
\hline 1062 & 22 & 18 & 20 & 22 & 90 & 95 & & & & 18 & 24 & 41 & 40 \\
\hline 10 B 2 & 98 & 102 & 20 & 42 & 76 & 86 & 804 & 125 & 139 & 18 & 44 & 15 & 19 \\
\hline 10102 & 119 & 118 & 20 & 62 & 49 & 45 & 824 & 158 & 180 & 18 & 64 & 29 & 27 \\
\hline 10122 & 41 & 42 & 20 & 82 & 44 & 43 & 844 & 69 & 64 & 18 & 84 & 106 & 118 \\
\hline 10142 & 109 & 117 & 201 & 102 & 44 & 48 & 8 64 & 61 & 64 & 18 & 104 & 37 & 42 \\
\hline 10162 & 73 & 73 & & & & & $8 \quad 84$ & 36 & 36 & & & & \\
\hline 10182 & 74 & 67 & 22 & 02 & 60 & 62 & 8104 & 27 & 25 & 20 & 04 & 51 & 53 \\
\hline 10202 & 32 & 25 & 22 & 22 & 27 & 25 & 8124 & 59 & 59 & 20 & 24 & 23 & 26 \\
\hline & & & 22 & 42 & 39 & 38 & 8144 & 44 & 38 & 20 & 44 & 20 & 25 \\
\hline
\end{tabular}

The following average interatomic distances calculated from the data in Table 4 are of particular interest:

$$
\begin{array}{lr}
\mathrm{Si}-\mathrm{O} & : 1.62 \pm 0.01 \AA \\
\mathrm{Al}-\mathrm{O} & : 1.75 \pm 0.02 \AA \\
\mathrm{Na}-\mathrm{O} & : 2.37 \pm 0.02 \AA \\
\mathrm{Na}-\mathrm{H}_{2} \mathrm{O} & : 2.38 \pm 0.03 \AA .
\end{array}
$$


Table 4. Interatomic distances and bond angles calculated from the final parameters

Interatomic distances in $\AA$

$\mathrm{SiO}_{4}$ tetrahedra:
$\mathrm{Si}_{\mathrm{I}}-\mathrm{O}_{1}$
$\mathrm{Si}_{\mathrm{I}}-\mathrm{O}_{\mathrm{V}}$
$\mathrm{Si}_{\text {II }}-\mathrm{O}_{\mathrm{II}}$
$\mathrm{Si}_{\text {II }}-\mathrm{O}_{\text {III }}$
$\mathrm{Si}_{\text {II }}-\mathrm{O}_{\mathrm{IV}}$
$\mathrm{Si}_{\mathrm{II}}-\mathrm{O}_{\mathrm{V}}$

\begin{tabular}{l|l}
$1.63 \pm 0.01$ & $\mathrm{O}_{\mathrm{I}}-\mathrm{O}_{\mathrm{I}}$ \\
$1.62 \pm 0.01$ & $\mathrm{O}_{\mathrm{I}}-\mathrm{O}_{\mathrm{V}}$ \\
$1.61 \pm 0.02$ & $\mathrm{O}_{\mathrm{V}}-\mathrm{O}_{\mathrm{V}}$ \\
$1.62 \pm 0.02$ & $\mathrm{O}_{\mathrm{II}}-\mathrm{O}_{\mathrm{III}}$ \\
$1.60 \pm 0.02$ & $\mathrm{O}_{\mathrm{II}}-\mathrm{O}_{\mathrm{IV}}$ \\
$1.64 \pm 0.02$ & $\mathrm{O}_{\mathrm{II}}-\mathrm{O}_{\mathrm{V}}$ \\
& $\mathrm{O}_{\mathrm{III}}-\mathrm{O}_{\mathrm{IV}}$ \\
& $\mathrm{O}_{\mathrm{III}}-\mathrm{O}_{\mathrm{V}}$ \\
& $\mathrm{O}_{\mathrm{IV}}-\mathrm{O}_{\mathrm{V}}$
\end{tabular}

$1.73 \pm 0.02$

$1.76 \pm 0.02$

$1.73 \pm 0.02$

$1.77 \pm 0.02$

Al一O II

Al $-\mathrm{O}_{\mathrm{IV}}$

$$
\begin{aligned}
& \mathrm{O}_{\mathrm{I}}-\mathrm{O}_{\mathrm{II}} \\
& \mathrm{O}_{\mathrm{I}}-\mathrm{O}_{\mathrm{III}} \\
& \mathrm{O}_{\mathrm{I}}-\mathrm{O}_{\mathrm{IV}} \\
& \mathrm{O}_{\mathrm{II}}-\mathrm{O}_{\mathrm{III}} \\
& \mathrm{O}_{\mathrm{II}}-\mathrm{O}_{\mathrm{IV}} \\
& \mathrm{O}_{\mathrm{III}}-\mathrm{O}_{\mathrm{IV}}
\end{aligned}
$$

$2.36 \pm 0.02$
$2.39 \pm 0.02$
$2.40 \pm 0.03$
$2.36 \pm 0.03$
$2.51 \pm 0.02$
$2.62 \pm 0.02$
$3.65 \pm 0.02$

$$
\begin{aligned}
& \mathrm{H}_{2} \mathrm{O}-\mathrm{O}_{\mathrm{I}} \\
& \mathrm{H}_{2} \mathrm{O}-\mathrm{O}_{\mathrm{II}} \\
& \mathrm{H}_{2} \mathrm{O}-\mathrm{O}_{\mathrm{III}} \\
& \mathrm{H}_{2} \mathrm{O}-\mathrm{O}_{\mathrm{IV}} \\
& \mathrm{H}_{2} \mathrm{O}-\mathrm{O}_{\mathrm{V}}
\end{aligned}
$$

$\mathrm{O}_{\mathrm{I}}-\mathrm{Si}_{\mathrm{I}}-\mathrm{O}_{\mathrm{I}}$

$\mathrm{O}_{\mathrm{I}}-\mathrm{Si}_{\mathrm{I}}-\mathrm{O}_{V}$

$\mathrm{O}_{\mathbf{I}}-\mathrm{Si}_{\mathbf{I}}-\mathrm{O}_{\mathrm{V}}$

$\mathrm{O}_{\mathrm{V}}-\mathrm{Si}_{\mathrm{I}}-\mathrm{O}_{\mathrm{V}}$

$\mathrm{O}_{\text {II }}-\mathrm{Si}_{\mathrm{II}}-\mathrm{O}_{\mathrm{III}}$

$\mathrm{O}_{\mathrm{II}}-\mathrm{Si}_{\mathrm{II}}-\mathrm{O}_{\mathrm{IV}}$

$\mathrm{O}_{\mathrm{II}}-\mathrm{Si}_{\mathrm{II}}-\mathrm{O}_{\mathrm{V}}$

$\mathrm{O}_{\mathrm{III}}-\mathrm{Si}_{\mathrm{II}}-\mathrm{O}_{\mathrm{IV}}$

$\mathrm{O}_{\mathrm{III}}-\mathrm{Si}_{\mathrm{II}}-\mathrm{O}_{\mathrm{V}}$

$\mathrm{O}_{\mathrm{IV}}-\mathrm{Si}_{\mathrm{II}}-\mathrm{O}_{\mathrm{V}}$

$\mathrm{Si}_{\mathrm{I}}-\mathrm{O}_{\mathrm{V}}-\mathrm{Si}_{\mathrm{II}}$

$\mathrm{Si}_{\mathrm{I}}-\mathrm{O}_{\mathrm{I}}-\mathrm{Al}$

$\mathrm{Si}_{\text {II }}-\mathrm{O}_{\mathrm{II}}-\mathrm{Al}$

$\mathrm{Si}_{\text {II }}-\mathrm{O}_{\mathrm{III}}-\mathrm{Al}$

\section{Bond angles}
$110.5^{\circ}$
$108.2^{\circ}$
$108.6^{\circ}$
$112.8^{\circ}$
$107.4^{\circ}$
$111.8^{\circ}$
$106.7^{\circ}$
$111.7^{\circ}$
$110.8^{\circ}$
$108.8^{\circ}$
$143.4^{\circ}$
$162.3^{\circ}$
$129.1^{\circ}$
$138.7^{\circ}$

$110.5^{\circ}$

$109.5^{\circ}$

$113.1^{\circ}$

$111.0^{\circ}$

$108.1^{\circ}$

$109.6^{\circ}$

$87.4^{\circ}$

$92.2^{\circ}$

$93.0^{\circ}$

$141.7^{\circ}$

$134.5^{\circ}$ 


\section{Discussion of the strueture}

The aluminosilicate framework of natrolite has several interesting features. The dehydration and cation exchange phenomena have long been attributed to the channels parallel to the $c$ axis (TAYLOR, 1934), which can easily be recognized in Fig. 2. The openings between neighboring chains (as shown in Fig. 1), however, provide an additional system of intersecting channels which are even more open. These channels have a minimum free diameter of $2.60 \AA$, whereas $2.08 \AA$ is obtained for the corresponding width of the established channels parallel to the $c$ axis. These data, however, do not represent limiting values for the size of exchangeable ions and molecules as in the case of rigid zeolite frameworks (e.g. BARRER and MEIER, 1958). The absence of a sieve action is due to the ability of the chains to rotate around the $c$ axis to some extent, which changes the size of the openings. The observed changes in the cell constants $a$ and $b$ of ion-exchanged natrolite samples (HEY and BANNISTER, 1933) provide evidence for the changes in the orientation of the chains. Even so, it appears unlikely that sorption and exchange diffusion processes should take place along the narrower and much longer channels parallel to the fiber axis as has been supposed. The same applies to the related structures of thomsonite and edingtonite where the difference in the openness of the established channels $(\| c)$ and the intersecting main channels $\left(\perp_{-} c\right)$ is even more striking.

The ordered arrangement of the $\mathrm{Al}$ and $\mathrm{Si}$ atoms first proposed by PadLING (1930) has been fully confirmed. It is noteworthy that any other distribution of the $\mathrm{Al}$ and $\mathrm{Si}$ atoms in the unit cell would not only lower the symmetry but would also lead to neighboring $\mathrm{AlO}_{4}$ tetrahedra with shared oxygen atoms requiring a bond of strength 0.5 for saturation. The requirements of the electrostatic valence rule, as well as extensive experimental evidence collected by Lozwenstein (1954), appear to rule out such an arrangement (even in the presence of polyvalent cations). It may also be shown that the $\mathrm{Al} / \mathrm{Si}$ ratio in natrolite cannot exceed the value of $2: 3$ in an ordered framework without Al- $-\mathrm{O}-\mathrm{Al}$ bonds ${ }^{4}$.

The average interatomic distances of $1.62 \pm 0.01 \AA$ for the $\mathrm{Si}-\mathrm{O}$ bond and $1.75 \pm 0.02 \AA$ for the $\mathrm{Al}-\mathrm{O}$ bond do not differ significantly from the values of $1.60 \pm 0.01 \AA$ and $1.78 \pm 0.02 \AA$ accepted by Sмiтt (1954). The $\mathrm{Si}-\mathrm{O}$ bond length is in full agreement with the

4 A detailed account of the $\mathrm{Al}-\mathrm{Si}$ distribution in fibrous zeolites will appear elsewhere. 
recent results of $1.62 \pm 0.01 \AA$ found in danburite, $\mathrm{CaB}_{2} \mathrm{Si}_{2} \mathrm{O}_{8}$, by JoHansson (1959) and $1.61 \pm 0.01 \AA$ in coesite, the high-pressure form of silica, as determined by ZOLTAI and BUERGER (1959). The somewhat low value for the $\mathrm{Al}-\mathrm{O}$ bond distance seems to suggest some substitution of $\mathrm{Al}$ atoms by $\mathrm{Si}$ as indicated by the result of the alkali determination. It should be noted, however, that the observed difference is within the limits of error and thus does not represent sufficient evidence for any substitution of $\mathrm{Al}$ by $\mathrm{Si}$. Moreover, the extensive analytical data reported by HeY and BANNisTer (1933) clearly indicate a fixed $\mathrm{Al} / \mathrm{Si}$ ratio of $2: 3$.

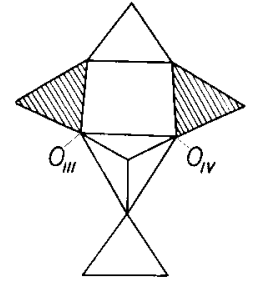

Fig. 4

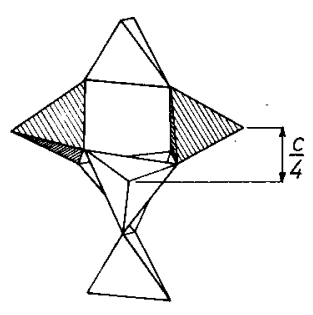

Fig. 4. Ideal and distorted natrolite chain.

Fig. 5. The arrangement of the sodium ions and water molecules in natrolite (slightly idealized).

The undistorted natrolite chain built up of regular tetrahedra of the established size (Sмiтh, 1954) has a repeat distance of $6.61 \AA$ which is in good agreement with the observed value of $c=6.60 \AA$. The oxygen atoms $\mathrm{O}_{\mathrm{II}}$ which provide the links for neighboring chains are at heights differing by $1.73 \AA$ in the undistorted chain. The value required by the structure is $\frac{c}{4}=1.65 \AA$, however, which should give rise to some distortion of the chains. The bond angles listed in Table 4 show that the tetrahedra undergo some deformation as a result of this requirement. The deviations from regularity of the tetrahedra are mostly within the limits of error of this determination, however. By far the most significant irregularity, on the other hand, lies in the difference of the $z$ parameters of $\mathrm{O}_{\mathrm{III}}$ and $\mathrm{O}_{\mathrm{IV}}(0.4997$ and $0.4666 \mathrm{in}$ the chain of Fig.4). This difference indicates twisted chains as illus- 
trated in Fig.4. The requirement mentioned above as well as the electrostatic charge distribution discussed below may account for the twisting of the chains. The $\mathrm{Si}-\mathrm{O}-(\mathrm{Si}, \mathrm{Al})$ bond angles in natrolite (Table 4 ) are within the range of about $130^{\circ}-160^{\circ}$ normally observed in such structures.

The arrangement of the cations and water molecules in the zeolite is shown in Fig. 5. Each sodium atom has four nearest neighbors, two oxygen atoms $\left(\mathrm{O}_{\mathrm{III}}\right.$ and $\left.\mathrm{O}_{\mathrm{IV}}\right)$ at an average distance of $2.37 \AA$ and two water molecules at an average distance of $2.38 \AA$. These interatomic distances are considerably shorter than the value of $2.6 \AA$ found in earlier investigations (PAULING, 1930; TAYLOR et al., 1933) and compare well with the value of $2.35 \AA$ for the ionic $\mathrm{Na}-\mathrm{O}$ bond length (Pauling, 1952). Two more oxygen atoms lie somewhat further away from the sodium atoms, the distances being 2.51 and $2.62 \AA$. These two oxygen atoms $\mathrm{O}_{\mathrm{II}}$ have also to be considered in the estimation of bond strengths contributed by the central $\mathrm{Na}^{+}$ion. The bond strength contributions by $\mathrm{Al}^{3+}$ and $\mathrm{Si}^{4+}$ to the $\mathrm{O}^{2-}$ ions of

Table 5. Electrostatic charge distribution around the sodium ions and water molecules

\begin{tabular}{|c|c|c|}
\hline Bond & $\begin{array}{c}\text { Interatomic } \\
\text { distance }\end{array}$ & $\begin{array}{c}\text { Estimated } \\
\text { bond strength }\end{array}$ \\
\hline $\mathrm{Na}--\mathrm{O}_{\mathrm{III}}$ & $2.36 \AA$ & 0.2 \\
\hline $\mathrm{Na}-\mathrm{O}_{\mathrm{IV}}$ & $2.39 \AA$ & 0.2 \\
\hline $\mathrm{Na}-\mathrm{H}_{2} \mathrm{O}(1)$ & $2.40 \AA$ & 0.2 \\
\hline $\mathrm{Na}-\mathrm{H}_{2} \mathrm{O}(2)$ & $2.36 \AA$ & 0.2 \\
\hline $\mathrm{Na}-\mathrm{O}_{\mathrm{II}}(1)$ & $2.51 \AA$ & 0.1 \\
\hline $\mathrm{Na}-\mathrm{O}_{\mathrm{II}}(2)$ & $2.61 \AA$ & 0.1 \\
\hline $\mathrm{H}_{2} \mathrm{O}-\mathrm{O}_{\mathrm{I}}$ & $2.86 \AA$ & 0.3 \\
\hline $\mathrm{H}_{2} \mathrm{O}-\mathrm{O}_{\mathrm{v}}$ & $2.99 \AA$ & 0.1 \\
\hline
\end{tabular}

the framework amount to 2.0 for $\mathrm{O}_{\mathrm{V}}$ and 1.75 for $\mathrm{O}_{\mathrm{I}}, \mathrm{O}_{\mathrm{II}}, \mathrm{O}_{\mathrm{III}}$, and $\mathrm{O}_{\mathrm{IV}}$. Estimated values for the strength of the $\mathrm{Na}-\mathrm{O}$ and $\mathrm{H}_{2} \mathrm{O}-\mathrm{O}$ bonds are listed in Table 5 and lead to bond strength totals of 2.1 for $O_{V}, 2.05$ for $O_{I}$, and 1.95 for $\mathrm{O}_{\mathrm{II}}, \mathrm{O}_{\mathrm{III}}$ and $\mathrm{O}_{\mathrm{IV}}$. A satisfactory local balance of charges is thus obtained.

The locations of the protons cannot be deduced with rigor. The interatomic distances between the water molecule and the surrounding oxygen atoms (Table 4) together with the electrostatic charge distribution just discussed, provide strong evidence that the water is linked to $\mathrm{O}_{\mathrm{I}}$ and $\mathrm{O}_{\mathrm{V}}$ by hydrogen bonds having a length of 2.85 and $2.99 \AA$ respectively.

The dehydration of the zeolite causes some serious disturbances in the local charge balance. It seems likely that the $\mathrm{Na}^{+}$ion will tend to move towards $\mathrm{O}_{\mathrm{I}}$ on dehydration in order to contribute to the charge balance on $\mathrm{O}_{I}$. The resultant increase in the $\mathrm{Na}-\mathrm{O}_{\mathrm{II}}$ and other bond distances would have to be counteracted by some changes in the orien- 
tation of the chains. A careful examination reveals that this would lead to a decrease in the cell constants $a$ and $b$. X-ray photographs of dehydrated crystals were found to be rather poor, unfortunately, owing to the tendency of the crystals to break up into bundles of fibers on dehydration. The photographs, nevertheless, revealed a small decrease in the cell constants $a$ and $b$ which was also observed by HeY and BANNISTER (1933). The shrinking of the natrolite lattice on dehydration thus appears to be a consequence of the requirements set forth by the electrostatic valence rule.

I would like to express my sincere thanks to Professor Linus Paulivg for suggesting this study, and for his kind interest and valuable advice during the course of this work. Thanks are also due to Drs. E. W. Hughes, G. Johansson, and W. Barclay Kamb for many helpful discussions. Finally, I wish to acknowledge the tenure of an Arthur Amos Noyes Fellowship.

\section{References}

R. M. BARRER and W. M. MEIER (1958), Structural and ion sieve properties of a synthetic crystalline exchanger. Trans. Farad. Soc. 54, 1074-1085.

J. Berghois, I. M. HaAnappex, M. Potters, B. O. Loopstra, C. H. MoGiLLAVRY and A. L. VEENENDAAI (1955), New calculations of atomic scattering factors. Acta Crystallogr. 8, 478-483.

F. HALLA und E. MEHU (1930), Das Raumgitter des Natroliths. Z. Kristallogr. $75,421-429$.

M. H. Hey and F. A. Bannister (1933), Studies on the zeolites III. Natrolite and metanatrolite. Min. Mag. 23, 243-289.

E. W. Hughes (1941), The crystal structure of melamine. J. Am. Chem. Soc. 63, 1737-1752.

G. Johansson (1959), A refinement of the crystal structure of danburite. Acta Crystallogr. 12, 522-525.

W. LoEwENSTEIN (1954), The distribution of aluminum in the tetrahedra of silicates and aluminates. Am. Mineral. 39, 92-96.

L. PAULING (1930), The structure of some sodium and calcium aluminosilicates. Proc. Nat. Acad. Sci. 16, $453-459$.

- (1952), The nature of the chemical bond. Oxford University Press, 2 nd ed.

J. V. Smiтh (1954), A review of the Al-O and Si-O distances. Acta Crystallogr. $7,479-481$.

W. H. TAYLOR (1934), The nature and properties of aluminosilicate framework structures. Proc. Roy. Soc. [London] A 145, 80-103.

-, C. A. MeEk and W. W. JACKSON (1933), The structures of the fibrous zeolites. Z. Kristallogr. 84, 373-398.

Y. Tomile and C. H. STAM (1958), Calculation of atomic scattering factors using Slater wave functions : sodium to calcium. Acta Crystallogr. 11, 126-127.

T. ZoltaI and M. J. BusRger (1959), The crystal structure of coesite, the dense, high-pressure form of silica. Z. Kristallogr. 111, 129-141. 